\title{
A heritability-based comparison of methods used to cluster 165 rRNA gene sequences into operational taxonomic units.
}

\author{
Matthew A Jackson ${ }^{\text {Corresp., }}{ }^{1}$, Jordana T Bell ${ }^{1}{ }^{\text {, }}$,Tim D Spector ${ }^{1}$, Claire J Steves ${ }^{1}$ \\ ${ }^{1}$ Department of Twin Research \& Genetic Epidemiology, King's College London, University of London, London, United Kingdom \\ Corresponding Author: Matthew A Jackson \\ Email address: matthew.jackson@kcl.ac.uk
}

A variety of methods are available to collapse 16S rRNA gene sequencing reads to the operational taxonomic units (OTUs) used in microbiome analyses. A number of studies have aimed to compare the quality of the resulting OTUs. However, in the absence of a standard method to define and enumerate the different taxa within a microbial community, existing comparisons have been unable to compare the ability of clustering methods to generate units that accurately represent functional taxonomic segregation. We have previously demonstrated heritability of the microbiome and we propose this as a measure of each methods' ability to generate OTUs representing biologically relevant units. Our approach assumes that OTUs that best represent the functional units interacting with the hosts' properties will produce the highest heritability estimates. Using 1750 unselected individuals from the TwinsUK cohort, we compared 11 approaches to OTU clustering in heritability analyses. We find that de novo clustering methods produce more heritable OTUs than reference based approaches, with VSEARCH and SUMACLUST performing well. We also show that differences resulting from each clustering method are minimal once reads are collapsed by taxonomic assignment, although sample diversity estimates are clearly influenced by OTU clustering approach. These results should help the selection of sequence clustering methods in future microbiome studies, particularly for studies of human host-microbiome interactions. 
1 A heritability-based comparison of methods used to cluster 16S rRNA gene sequences into

2 operational taxonomic units.

3

4 Matthew A. Jackson ${ }^{1}$, Jordana T. Bell ${ }^{1}$, Tim D. Spector ${ }^{1}$, Claire J. Steves ${ }^{1}$

5

6 1. Department of Twin Research and Genetic Epidemiology, King's College London, London, $7 \mathrm{UK}$

8

9 Corresponding Author:

10

11 Matthew Jackson

12 Department of Twin Research \& Genetic Epidemiology,

13 King's College London,

14 St Thomas' Hospital Campus,

153 rd \& 4th Floor South Wing Block D,

16 Westminster Bridge Road,

17 London,

18 SE1 7EH

19 Phone: +44 (0) 2071881508

20 Email: matthew.jackson@kcl.ac.uk

21 


\section{ABSTRACT}

23 A variety of methods are available to collapse 16S rRNA gene sequencing reads to the

24 operational taxonomic units (OTUs) used in microbiome analyses. A number of studies have

25 aimed to compare the quality of the resulting OTUs. However, in the absence of a standard

26 method to define and enumerate the different taxa within a microbial community, existing

27 comparisons have been unable to compare the ability of clustering methods to generate units that

28 accurately represent functional taxonomic segregation. We have previously demonstrated

29 heritability of the microbiome and we propose this as a measure of each methods' ability to generate OTUs representing biologically relevant units. Our approach assumes that OTUs that best represent the functional units interacting with the hosts' properties will produce the highest

32 heritability estimates. Using 1750 unselected individuals from the TwinsUK cohort, we compared 11 approaches to OTU clustering in heritability analyses. We find that de novo clustering methods produce more heritable OTUs than reference based approaches, with VSEARCH and SUMACLUST performing well. We also show that differences resulting from each clustering method are minimal once reads are collapsed by taxonomic assignment, although sample diversity estimates are clearly influenced by OTU clustering approach. These results should help the selection of sequence clustering methods in future microbiome studies, particularly for studies of human host-microbiome interactions. 


\section{INTRODUCTION}

43

44 The field of microbiome research has seen rapid expansion this last decade (Jones 2013). One of

45 the techniques most frequently used to profile microbial communities is 16S rRNA gene

46 sequencing, where PCR amplification of variable marker regions is used to determine a sample's

47 microbial composition (Pace 1997). The taxonomic resolution of sequence variation across a

48 marker region is limited both biologically and technically, because sequence divergence may not 49 represent wider biological divergence between taxa (Stackebrandt \& Goebel 1994; Mignard \&

50 Flandrois 2006), and sequencing errors introduce artificial divergence (Huse et al. 2010; Schloss

51 et al. 2011). As a result, it is not necessarily useful to enumerate every unique sequence

52 observed, particularly given that samples may contain hundreds of thousands of unique reads. To

53 simplify analyses, reads within a 16S rRNA gene dataset are typically collapsed into operational

54 taxonomic units (OTUs). This is carried out based on sequence similarity between reads.

55 Convention is typically to group reads that share at least $97 \%$ identity, which is considered

56 "species" level. Although collapsing can be carried out to any threshold and there is no clear

57 definition of what constitutes a bacterial species.

58

59 A variety of methods are available to collapse 16S data to OTUs (Edgar 2010; Edgar 2013;

60 Rognes et al. 2016; Mercier et al. 2013; Mahé et al. 2014; Schloss \& Handelsman 2005; Eren et

61 al. 2014), often implemented within software wrappers such as QIIME and Mothur (Caporaso et

62 al. 2010; Schloss et al. 2009). One of the main divides in approaches is whether experimental

63 sequences are clustered against a reference database of sequences (Liu et al. 2008), termed

64 closed reference clustering (Navas-Molina et al. 2013), or solely clustered within the

65 experimental data itself, generating what are termed de novo OTUs (Schloss \& Handelsman

66 2005; Navas-Molina et al. 2013). Closed reference clustering is computationally more efficient

67 given that each sequence should maximally only be compared against each reference sequence,

68 whereas de novo clustering could require pair-wise comparisons between all experimental reads.

69 Closed reference approaches also facilitate comparisons between datasets as OTUs can be

70 defined and matched based on their reference sequences; however, reads which do not match any

71 reference sequences will be discarded. De novo clustering does not have this limitation and

72 includes all experimental reads in resultant OTUs, which may better represent rare and novel 
73 taxa (Navas-Molina et al. 2013). A third approach, termed open-reference clustering, aims to

74 capitalise on the benefits of both approaches by first clustering experimental sequences against a

75 reference followed by de novo clustering of discarded sequences (Navas-Molina et al. 2013).

76

77 Once a reference or de novo based approach has been selected, a number of different algorithms

78 can be used to cluster sequences by similarity (Schloss \& Handelsman 2005; Caporaso et al.

79 2010; Edgar 2010; Edgar 2013; Rognes et al. 2016; Mercier et al. 2013; Mahé et al. 2014; Eren

80 et al. 2014). Linkage based methods calculate pairwise distances between all sequences allowing

81 hierarchical clustering to OTUs (Schloss \& Handelsman 2005). There are also multiple greedy

82 algorithms available, which aim to reduce computation time using heuristic approaches to

83 finding optimal groups without calculating all possible distances (Edgar 2010; Edgar 2013;

84 Rognes et al. 2016). Furthermore, there have been a number of methods proposed to summarise

$8516 \mathrm{~S}$ data without using a predetermined global similarity threshold. These include simply using

86 de-replicated sequences (reads collapsed by 100\% similarity), defining OTUs by inherent

87 separation within the dataset using local rather than global cut-offs (Mahé et al. 2014), and

88 splitting reads into groups based on sequence entropy at each position in aligned reads (Eren et

89 al. 2014).

90

91 With the range of available approaches to OTU picking some comparative metric is required to

92 assess their performance. Previously, clustering algorithms have been compared based on a

93 number of metrics including: their computational efficiency (Edgar 2010; Kopylova et al. 2014;

94 Chen et al. 2013); the number of OTUs they produce (Schmidt et al. 2014; Kopylova et al. 2014;

95 Chen et al. 2013); the accuracy of the similarity between sequences within their OTUs (Westcott

96 \& Schloss 2015; Schloss et al. 2011; Schloss 2016); their ability to handle sequencing artefacts

97 (Edgar 2013); their reconstruction of simulated data sets (Kopylova et al. 2014; Chen et al.

98 2013); the similarity between method outputs (Schmidt et al. 2014; Kopylova et al. 2014); and

99 the reproducibility of their clustering within subsets of the same data (He et al. 2015). However,

100 the optimal approach between de novo and reference clustering, and the different clustering

101 algorithms is dependent on which measure of quality is considered.

102 
103 As there is no accepted standard for definition and enumeration of microbial taxa in a

104 community, existing comparison metrics have exclusively dealt with technical aspects of

105 clustering. It is not clear which of these metrics is most important in determining a methods

106 ability to generate OTUs most representative of the biological units underlying microbial

107 community structure. Here we suggest heritability as a measure of the biological relevance of

108 OTUs.

109

110 Heritability quantifies the percentage of phenotypic variation that is attributable to genetic

111 variability. Twin studies are a well-established method for estimating heritability. These

112 compare the correlation of phenotypes within monozygotic (MZ) twin pairs whom share

113 identical nuclear DNA, to the correlations within dizygotic (DZ) pairs whom on average share

114 half their genetic material. Variation in a phenotype can then be apportioned into variation due to

115 genetic factors, which are shared by twins to a varying degree, based on zygosity and to

116 environmental factors, which are not shared by twins (Franic et al. 2012; Boomsma et al. 2002).

117

118 TwinsUK is a long established cohort of unselected British twins (Moayyeri et al. 2013). 16S

119 rRNA gene sequencing of faecal samples from the cohort has been used to demonstrate

120 heritability of the microbiome (Goodrich et al. 2014; Goodrich et al. 2016), and to identify a

121 number of phenotype-microbiome associations (Jackson et al. 2016; Jackson et al. 2015; Barrios

122 et al. 2015). Under the assumption that some heritability within the microbiome is acting at the

123 level of individual taxa-host interactions, we propose that the heritability of OTUs is

124 representative of their ability to summarise the underlying biological units within a microbial

125 community.

126

127 Here we compare heritability estimates of 11 different methods of summarising $16 \mathrm{~S}$ reads from

1281750 faecal samples of $473 \mathrm{MZ}$ and $402 \mathrm{DZ}$ twin pairs. Overall, we find that de novo clustering,

129 regardless of algorithm, consistently produces more heritable OTUs than reference based

130 approaches, with VSEARCH and SUMACLUST producing the highest heritability estimates

131 from those considered. No difference in heritability was observed once OTUs had been collapsed

132 by taxonomic assignment. We also find that clustering method can influence relative sample

133 diversity, dependant on the diversity metric used. These results should provide guidance to 
134 researchers in selecting the appropriate approach to OTU picking, in particular in studies

135 investigating human host-microbiome interactions.

136

\section{METHODS}

138

139

\section{Faecal Sampling and 16S rRNA gene Sequencing}

140 Analyses were carried out using 16S rRNA gene sequencing reads from a subset of published

141 data from the TwinsUK cohort. Sample collection, DNA extraction and sequencing have

142 previously been reported (Goodrich et al. 2014). In brief, twins produced the sample at home,

143 which was then kept refrigerated and/or on ice before freezing at $-80^{\circ} \mathrm{C}$ in the TwinsUK

144 laboratory at King's College London. Frozen samples were then shipped to Cornell University

145 where extracted DNA from samples was PCR amplified over the V4 variable region of the 16S

146 gene. The resulting amplicons were multiplexed and sequenced using the Illumina MiSeq

147 platform to generate 250bp paired-end reads. Ethical approval for microbiota studies within

148 TwinsUK were provided by the NRES Committee London - Westminster (REC Reference No.:

149 EC04/015). All participants provided written consent.

150

\section{Pre-processing of sequencing reads}

152 Paired reads were joined using fastqjoin, within QIIME (Caporaso et al. 2010), discarding reads

153 without a minimum overlap of 200nt and those containing ambiguous bases. Joined reads were

154 de-multiplexed also removing barcodes. The data were filtered to only include the subset of 1750

155 samples from the $473 \mathrm{MZ}$ and $402 \mathrm{DZ}$ complete twin pairs used in these analyses. Within this

156 set, there were 158635772 reads with an average of 91170 reads per sample. These were split

157 per sample and de novo chimera checking carried out on each individually using USEARCH de

158 novo chimera detection in QIIME with a no vote weight of 7 (Edgar et al. 2011; He et al. 2015).

159 This identified an average of 8471 chimeric reads per sample all of which were removed.

160 Sample reads were then concatenated to one file and all sequences $<252 \mathrm{nt}$ or $>253 \mathrm{nt}$ in length

161 discarded ( $<1 \%$ of reads) (Kozich et al. 2013). After chimera removal and length filtering, the

162 final data set contained 142307280 reads across all samples. This fasta file was used as the

163 input for all $16 \mathrm{~S}$ collapsing approaches. 
165 These reads and associated metadata, covering a larger selection of samples and twins than the

166 subset described here, are available from the European Nucleotide Archive (ENA) from the

167 study with accession number ERP015317 (http:/www.ebi.ac.uk/ena/data/view/ERP015317)

168 (Goodrich et al. 2016).

169

170 Clustering of 16S rRNA gene sequencing reads

171 All threshold based OTU clustering approaches and Swarm were implemented using QIIME

172 1.9.0 (Caporaso et al. 2010; Mahé et al. 2014). VSEARCH de novo clustering was implemented 173 within the QIIME wrappers using an alias to run VSERARCH in place of USEARCH (Rognes et

174 al. 2016; Edgar 2010). VSEARCH is not restricted to the same memory limitations as the free

175 version of USEARCH, enabling its use across our whole data set. It also accepts the same

176 commands for de novo clustering so required no alterations to the QIIME wrapper. Where a

177 reference was required, the Greengenes reference and taxonomy version 13_8 was used

178 (DeSantis et al. 2006). De-replicated sequences were generated using VSEARCH (Caporaso

179 2015). Minimum entropy decomposition (MED) was run from scripts within the oligotyping

180 pipeline using default parameters (Eren et al. 2014; Eren et al. 2013). An overview of how each

181 clustering method works, the clustering pipeline, and complete commands used for each

182 clustering procedure can be found in Supplementary Material S1.

183

\section{Heritability analyses}

185 Heritability of microbiome traits was calculated in a manner similar to as previously reported

186 (Goodrich et al. 2014). Estimates were calculated for OTUs found in at least 50\% of samples as

187 OTU absence, which skews the distribution of abundances, would be less influential on model

188 fitting. A pseudo count of 1 was added to all OTUs to remove absent data in the resultant OTU

189 tables of each clustering approach. Counts were converted to within sample relative abundances

190 and tables subset to only include OTUs found in at least 50\% of samples (prior to the addition of

191 pseudo counts). The powerTransform package in $\mathrm{R}$ was used to estimate a Box-Cox transform

192 lambda producing approximately normally distributed residuals from a linear model with OTU

193 abundance as a response and gender, age, sequencing run, sequencing depth, how the sample was

194 collected, and the technician who loaded and extracted the DNA as predictors. This was carried

195 out for each OTU and the transformed residuals used in heritability estimation. 
197 Estimates were found by fitting OTU abundances to a twin-based ACE model. This estimates 198 narrow-sense heritability (the heritability due to additive genetic effects - A) on the assumption 199 that variance resulting from shared environment (common environment $-\mathrm{C}$ ) is equal in $\mathrm{MZ}$ and 200 DZ twins, with remaining variance attributed to environmental influences unique to individuals 201 (E) (Franic et al. 2012). Maximum likelihood estimates were found by structural equation 202 modelling using OpenMX in R (R Development Core Team 2009; Boker et al. 2011).

203 Heritability estimates for collapsed taxonomic traits were calculated in the same manner as for 204 OTUs.

205

206 Between method comparisons of OTU heritability and other distributions were carried out in R 207 using pairwise Mann-Whitney U tests using Benjamini-Hochberg FDR correction to account for 208 multiple testing.

209

\section{Alpha diversity calculation and taxonomic assignment}

211 Each complete OTU table was rarefied to 10000 sequences 25 times. Alpha diversity calculation 212 was carried out on each rarefied table for each method using Simpson, Shannon, Chao1 and raw 213 OTU count metrics, with final diversity values taken as the mean across all rarefactions. Alpha 214 diversity estimates were compared using Mann-Whitney U tests to contrast absolute values 215 between methods and Kendall rank correlations to compare sample rankings between methods.

217 For each clustering method, except closed reference, representative sequences were selected as 218 the most abundant read within each OTU. These were then used to assign taxonomy against the 219 Greengenes 13_8 database with a 97\% similarity threshold using the UCLUST method in the assign taxonomy script of QIIME. OTU tables were collapsed based on taxonomic assignment at

221 all levels from genus to phylum. Differences in heritability of taxa between methods were 222 compared using a generalised linear model in $\mathrm{R}$, to determine the ability of taxonomic 223 assignment and clustering method to predict heritability estimates as the response variable. This

224 was carried out across all taxonomic levels considering all taxa that were found across all 11 225 clustering approaches. 


\section{RESULTS}

228

229

\section{De novo clustering produces more heritable OTUs than closed reference clustering}

230 16S microbiome profiles were available for $473 \mathrm{MZ}$ and $402 \mathrm{DZ}$ pairs within previously reported

231 data. Joined paired end read data were revisited and chimeric sequences removed on a per

232 sample basis. Total read data across all 1750 samples was then clustered using de novo, closed

233 reference, and open reference approaches using the UCLUST algorithm (Edgar 2010), the

234 current default in QIIME, to form OTUs with a threshold similarity of 97\%. The resultant OTU

235 tables are summarised in Supplementary Table S2. De novo clustering produced more OTUs

236 than closed reference and as a result, a more sparsely distributed OTU table. Open reference

237 picking was an intermediate of the two approaches as might be expected.

238

239 Across all three methods the A, C, and E estimates were within the range expected from previous

240 reports within the cohort (Goodrich et al. 2014, Goodrich et al. 2016). De novo clustering

241 produced OTUs with significantly higher ( $\mathrm{q}=0.017)$ heritability $(\mathrm{A})$ estimates than closed

242 reference clustering (Figure 1A). De novo heritability estimates were also higher than those of

243 open reference OTUs although the difference was non-significant. There were no significant

244 differences in the distributions of $\mathrm{C}$ estimates between any methods. De novo clustering

245 produced OTUs with significantly lower E estimates than both closed $(\mathrm{q}=0.02)$ and open

246 reference $(\mathrm{q}=0.003)$ approaches.

248 Whilst significant, the difference in OTU heritability estimates was only moderate. The mean of

249 the de novo A estimates was 1\% higher than that of the closed reference clustered OTUs.

250 However, the distribution of A, C, and E estimates were also divergent, as shown in Figure 1B.

251 Closed reference A estimates displayed a bimodal distribution with OTUs either having no or

252 little heritability with fewer highly heritable units. De novo clustering produced units of higher

253 heritability whose estimates were more evenly distributed. Open reference clustering displayed

254 features of both distributions resulting in higher levels of moderately heritable OTUs.

255

256 VSEARCH and SUMACLUST produce more heritable de novo OTUs than UCLUST 
257 As de novo clustering produced the most heritable OTUs using UCLUST, we aimed to

258 determine the influence of using alternative threshold based algorithms for clustering. Linkage

259 based clustering approaches were not considered as it was unfeasible to generate distance

260 matrices between the large number of unique reads within the data set. OTUs were clustered at

$26197 \%$ similarity using two alternate greedy algorithms within QIIME - VSEARCH and

262 SUMACLUST (Rognes et al. 2016; Mercier et al. 2013). The open-source algorithm VSEARCH

263 was used in place of the QIIME default USEARCH to overcome the memory limitations of its

264 free version. VSEARCH has previously been shown to match or outperform USEARCH in terms

265 of accuracy (Westcott \& Schloss 2015). Clustering with VSEARCH was carried out using both

266 distance and abundance options as tiebreak assignments. The resultant OTU tables are

267 summarised in Supplementary Table S2.

268

269 There were no significant differences in the mean magnitudes of the A, C, or E estimates

270 between all four methods tested (Figure 2A). The distributions of estimates were very similar in

271 the SUMACLUST, and both VSEARCH approaches (Figure 2B). UCLUST OTUs contained a

272 higher proportion of A estimates falling between 0.05 and 0.15 , with the other methods

273 containing higher proportions of more heritable OTUs. The VSEARCH methods had more

274 OTUs with high heritability estimates (0.35-0.4), with the distance tiebreaker based method

275 producing slightly fewer. SUMACLUST produced the most heritable OTU. Overall, all de novo

276 algorithms produced estimates higher than the UCLUST reference based approaches at a

277 threshold of 97\% similarity, with SUMACLUST and VSEARCH approaches producing more

278 heritable OTUs than UCLUST.

279

280 Clustering at higher thresholds and other alternatives to clustering

281 We aimed to investigate the use of more stringent thresholds repeating VSEARCH abundance

282 based clustering with identity thresholds of 98 and 99\%, and simply de-replicating the

283 sequences, the equivalent of a $100 \%$ threshold. We also clustered sequences using two

284 approaches that do not rely on a sequence identity threshold - MED and Swarm (described in

285 Supplementary Methods S1)(Eren et al. 2014; Mahé et al. 2014). Of the thresholds, 97\%

286 produced the most heritable OTUs (Figure 3A), whose distribution of A estimates was

287 significantly different to those of the $99(\mathrm{q}=0.02)$ and 100\% (q=0.0001) cut-off OTUs (Figure 
288 3B). As the percentage identity increased from $97 \%$ through to $100 \%$ the distribution of A

289 estimates became less continuous, with small groups of units with high heritability and much

290 larger numbers with low heritability. This suggests that in some instances, the heritability

291 estimate of an OTU clustered at 97\% identity may be driven by an individual, highly heritable

292 sequence; as opposed to the accumulative effects of the variance across all its reads.

293

294 MED produced very few units in total (Supplementary Table S2). However given this broad

295 level of summary, which is comparable to that of closed reference clustering, the resultant units

296 A estimates were not significantly different to VSEARCH OTUs clustered at the 97\% level.

297 Similarly, the heritability of OTUs resulting from clustering by Swarm had heritability's within

298 the range of the VSEARCH methods, however the distribution of A estimates more closely

299 resembled OTU clustering at a threshold of $99 \%$.

300

301

302

De novo clustering at $97 \%$ generates more heritable OTUs than reference-based

approaches when considering only heritable units

303 The power of a twin study to detect and accurately estimate the additive genetic variance of a

304 trait is limited by the total number of pairs and the proportion of MZ twins considered (Visscher

305 2004). As noise in the A estimates for non and low heritability traits may influence the overall

306 distribution, we compared A estimate distributions across all previously clustered techniques

307 considering only heritable OTUs - those with A estimates greater than the mean of all OTUs

$308(8 \%)$ and with a lower $95 \%$ confidence interval of at least $1 \%$ (Figure 4 ). When only

309 considering the most heritable OTUs, the majority of de novo based approaches produced units

310 with higher heritability estimates than the reference-based approaches. VSEARCH AGC

311 clustering at 97 and 98\%, and DGC clustering at 97\% produced significantly higher estimates

312 than closed reference UCLUST. As did SUMACLUST de novo clustering (97\% identity), which

313 also produced units with significantly higher heritability than those produced by open reference

314 based clustering. De novo clustering at higher sequence identity thresholds (99 and 100\%)

315 produced OTUs with significantly lower estimates than SUMACLUST at 97\%.

316

317 Differences resulting from clustering approach are not apparent after collapsing by 
319 The ability of a technique to generate OTUs representing fine scale biological units may be less

320 important for studies aiming to identify effects at higher taxonomic levels. To determine if

321 choice of OTU clustering approach significantly effected the ability to generate representative

322 taxa we collapsed each OTU table at all taxonomic levels from genus to phylum, and estimated

323 the heritability of taxa at each level (Supplementary Table S3). We then investigated the ability

324 of taxonomic assignments and clustering methods to predict taxa heritability estimates. We

325 found that assignments to 150 of the 168 taxa found across all 11 methods were significant

326 predictors of heritability, however none of the clustering methods had a significant effect. This

327 suggests that from genus through to higher-level taxonomic summaries, there is sufficient

328 collapsing of reads that the previously observed differences in OTU clustering are not apparent.

\section{Alpha diversity measures are influenced by clustering approach}

331 As the largest difference observed between methods was the number of OTUs generated, we 332 aimed to determine the influence of clustering approach on alpha diversity estimates. The 333 absolute values of sample diversity estimates were significantly different between almost all 334 methods of clustering for all four diversity estimates considered (Figure 5). In particular, the 335 values of OTU count and Chao1 (richness measures influenced by rarer OTUs) were much

336 higher in the de-replicated (or 100\% identity) sequences. These results show that absolute

337 diversity levels are not comparable between methods over the same rarefied data.

338

339 To determine if these differences would influence comparative diversity analyses, we measured

340 the rank based correlation between methods for each diversity metric (Figure 6). For both the

341 Shannon and Simpson metrics the diversity rankings were highly correlated $(\tau>0.6$, mean $=$

342 0.83) between all methods. However, when using the Chao1 and OTU count metrics there was a

343 reduced correlation between diversity rankings. In particular, the closed reference and MED

344 approaches were poorly correlated with de novo based approaches. This is likely due to under

345 representation of rare sequences as both of these methods discard reads. Our results show that

346 clustering approach can influence the relative diversities between samples in a study dependant

347 on the diversity measure used. This may be particularly important in the interpretation of

348 diversity association analyses, where use of a closed reference approach could produce different

349 results to the use of de novo clustering. 


\section{DISCUSSION}

352

353 Here we propose and demonstrate the use of heritability estimates as a novel approach to

354 methodological comparisons. There is an established taxa dependent variability in the heritability

355 of the gut microbiome (Goodrich et al. 2014). Heritability estimates aim to quantify the

356 percentage of a trait's variation that is due to the influence of host genetics. Given that bacteria

357 within the microbiome contain a range of functional properties, determined by their own

358 genetics, we assume that the heritability of an OTU is driven by a specific bacteria-host

359 interaction. By this logic, we would expect the OTU clustering approach that best groups reads

360 sourced from bacterial units with similar functional properties to produce OTUs with the highest 361 heritability estimates.

362

363 Using the distribution of heritability estimates as a measure of biological representation, we have 364 demonstrated that de novo clustering produces OTUs that are more representative of functional 365 microbial units than reference based approaches. We have also shown that within the various 366 algorithms considered VSEARCH and SUMACLUST produced the most representative OTUs. 367 Within our comparison of clustering thresholds, we found that $97 \%$ sequence identity produced 368 the most heritable units when compared to more stringent cut-offs. We have shown that these 369 effects are only applicable at the OTU level, as clustering approach does not significantly 370 influence the heritability estimates of collapsed taxonomies. Finally, we have demonstrated that 371 choice of clustering approach can effect both absolute and relative diversity measures with 372 implications for comparisons across microbial studies.

373

374 The aim of OTU clustering is to group sequences based on sequence similarity. Our comparisons 375 are based on the assumption that the genetic relatedness between $16 \mathrm{~S}$ reads is related to the 376 functional similarity between their bacterial sources. In this way, a clustering method that best 377 groups reads with similar sequence will also groups reads from bacteria with similar functional 378 relationships to the host. These methods should therefore produce the highest heritability 379 estimates, as they will produce less noise in the variance of OTU abundances due to incorrectly 380 grouped read counts. Whilst this may not provide an accurate quantification of the quality of 
381 sequence identity within OTUs (as provided by existing methods discussed below), it does

382 provide a measure of the functional representation of the units. For example, in our data the

383 OTUs clustered with 99 and 100\% identity thresholds produced lower heritability estimates.

384 Suggesting that $97 \%$ is the best threshold to generate units that represent functional units within

385 the microbiome. A methods ability to represent functional units is arguably of more importance

386 than genetic accuracy. Particularly for studies in areas such as human microbiome research

387 where the goal is often to identify the functional roles of microbes in human health.

389 Recently, four studies were published that each compared multiple OTU clustering approaches

390 (He et al. 2015; Kopylova et al. 2014; Westcott \& Schloss 2015; Schloss 2016). The first used

391 the stability of sequence assignments within subsets of the same data sets as a measure of

392 quality, finding that reference based approaches outperformed de novo clustering (He et al.

393 2015). The heritability comparisons presented here do not reflect these findings, suggesting that

394 stability does not relate to functional representation. However, stability may be an important

395 consideration for studies comparing across data sets. Our findings also suggest that reference

396 based approaches would be sufficient when analyses are only concerned with collapsed

397 taxonomies.

398

399 Two studies have compared clustering methods using Matthew's correlation coefficient (MCC)

400 to quantify their accuracy in clustering sequences sharing 97\% sequence identity (Westcott \&

401 Schloss 2015; Schloss 2016). They found that de novo clustering produced more accurate OTUs

402 than reference based approaches (Westcott \& Schloss 2015), and that VSEARCH and

403 SUMACLUST out performed Swarm in terms of OTU accuracy (Schloss 2016). The differences

404 between reference and de novo OTUs in our heritability estimates, whilst moderate, were

405 significant and broadly agreed with these observations. This suggests that accuracy is also

406 representative of the biological representation of OTUs. This might be expected under the

407 assumption that sequence similarity, at least in part, reflects functional similarity.

408

409 Kopylova et al. compared a number of clustering methods using a variety of measures from 410 recapitulation of simulated data, to inter-method correlations (Kopylova et al. 2014). Within the 411 methods considered here, they found that Swarm, SUMACLUST and UCLUST, performed 
412 equally well at reconstructing expected taxonomies from simulated data but differed in the 413 number of OTUs produced and subsequently produced different absolute diversities, a finding 414 also described by Schmidt et al. (Schmidt et al. 2014). Differences in absolute measures would 415 be expected given the variation in OTU numbers between methods. We have also shown that 416 these differences can influence the relative diversity rankings between samples and suggest 417 caution in the interpretation of comparative diversity analyses when using closed reference 418 clustering and community richness metrics.

420 Overall, across previous comparisons of greedy clustering algorithms in combination with the 421 heritability results we have presented here, VSEARCH and SUMACLUST seem to produce the 422 best combination of accuracy, stability and heritability. We would therefore recommend either of 423 these approaches for de novo clustering. SUMACLUST and USEARCH are currently available 424 within QIIME. VSEARCH has recently been implemented within Mothur (Westcott 2016), and 425 QIIME 2 will integrate VSEARCH for OTU clustering and de-replication (Greg Caporaso, 426 personal communication, $15^{\text {th }}$ April 2016). Based on our threshold comparisons a similarity cut427 off of 97\% appears optimal, however this threshold may be specific to VSEARCH application to 428 faecal samples as optimal thresholds can vary by the complexity of the microbial communities 429 under investigation and the method used (Chen et al. 2013).

430

431 Whilst we tried to include the most frequently used approaches, our study is not comprehensive.

432 We restricted the majority of our comparisons to clustering algorithms that were available within 433 the QIIME pipeline; however, even in this respect, our comparison was not exhaustive. There are 434 further reference based clustering algorithms such as BLAST and SortMeRNA that were not 435 considered (Camacho et al. 2009; Kopylova et al. 2012), and de novo approaches such as 436 USEARCH and CD-HIT (Edgar 2010; Li \& Godzik 2006). We chose to implement clustering 437 via QIIME as it is one of the most widely used methods to generate OTUs and provided stability 438 in other areas of the processing pipeline, such as taxonomic assignment, which improved 439 comparability. However, QIIME does not implement all OTU clustering algorithms and all of 440 those compared here can also be run independently of QIIME, with a number of them having 441 newer versions available that could influence clustering. Our comparison is also limited by the 442 exclusion of linkage-based approaches, as typically implemented using the Mothur pipeline 
443 (Schloss et al. 2009). These were not considered in our comparison due to the high

444 computational burden of generating the pair-wise sequence distance matrices that these methods

445 require. Computing time and memory limits were met even when applying additional sequence

446 filtering or restricting distance calculation by taxonomy (Kozich et al. 2013). Previous MCC

447 accuracy comparisons showed that average based linkage clustering were as or more accurate

448 than the best de novo approaches dependent on the dataset considered (Schloss 2016). Given the

449 reflection between the MCC and heritability results we might speculate that average linkage

450 based approaches could produce biologically relevant units equivalent to the de novo algorithms 451 we considered.

452

453 Our comparisons are further limited as we have only considered sequencing from human faecal 454 samples of a single population. A sufficiently large sample is required to determine heritability 455 estimates for moderately heritable traits (Martin et al. 1978), however clustering and analysis of 456 data on this scale is time consuming and computationally intensive making it non-trivial to 457 incorporate additional data. There are also few twin microbiome data sets available at the scale 458 of TwinsUK. It is known that existing measures of clustering quality can be data set dependent 459 (Schloss 2016; Chen et al. 2013; Kopylova et al. 2014). Therefore, our results may not be 460 applicable to non-faecal samples. However, they should be of particular relevance when 461 462

463 In conclusion, heritability analyses can be used to provide a measure of the quality of the 464 functional representation of OTUs. This may be used for additional guidance in selecting an 465 appropriate clustering approach in combination with the other comparative metrics available, 466 although the optimum method will be largely dependent on each studies experimental and 467 analytical requirements.

468

\section{ACKNOWLEDGEMENTS}

470 We would like to thank Julia Goodrich, Andrew Clark, and Ruth Ley of the Department of 471 Molecular Biology and Genetics at Cornell University, our collaborators on the collection, 472 processing, and analysis of the TwinsUK 16S gut microbiome data, whom provided guidance 473 and comments on this manuscript. 


\section{REFERENCES}

475

476

477

478

479

480

481

482

483

484

485

486

487

488

489

490

491

492

493

494

495

496

497

498

499

500

501

502

503

504

505

506

507

508

509

510

Barrios C., Beaumont M., Pallister T., Villar J., Goodrich JK., Clark A., Pascual J., Ley RE., Spector TD., Bell JT., Menni C. 2015. Gut-Microbiota-Metabolite Axis in Early Renal Function Decline. PLOS ONE 10:e134311. DOI: 10.1371/journal.pone.0134311.

Boker S., Neale M., Maes H., Wilde M., Spiegel M., Brick T., Spies J., Estabrook R., Kenny S., Bates T., Mehta P., Fox J. 2011. OpenMx: An Open Source Extended Structural Equation Modeling Framework. Psychometrika 76:306-317. DOI: 10.1007/s11336-010-9200-6.

Boomsma D., Busjahn A., Peltonen L. 2002. Classical twin studies and beyond. Nature Reviews Genetics 3:872882. DOI: $10.1038 /$ nrg932.

Camacho C., Coulouris G., Avagyan V., Ma N., Papadopoulos J., Bealer K., Madden TL. 2009. BLAST+: architecture and applications. BMC Bioinformatics 10:421. DOI: 10.1186/1471-2105-10-421.

Caporaso JG., Kuczynski J., Stombaugh J., Bittinger K., Bushman FD., Costello EK., Fierer N., Peña AG., Goodrich JK., Gordon JI., Huttley G a., Kelley ST., Knights D., Koenig JE., Ley RE., Lozupone C a., McDonald D., Muegge BD., Pirrung M., Reeder J., Sevinsky JR., Turnbaugh PJ., Walters W a., Widmann J., Yatsunenko T., Zaneveld J., Knight R. 2010. QIIME allows analysis of high-throughput community sequencing data. Nature Methods 7:335-336. DOI: 10.1038/nmeth.f.303.

Caporaso JG. 2015.VSEARCH-based sequence dereplication through generation of a biom table. Available at https://gist.github.com/gregcaporaso/f3c042e5eb806349fal8 (accessed April 20, 2016).

Chen W., Zhang CK., Cheng Y., Zhang S., Zhao H. 2013. A Comparison of Methods for Clustering 16S rRNA Sequences into OTUs. PLoS ONE 8:e70837. DOI: 10.1371/journal.pone.0070837.

DeSantis TZ., Hugenholtz P., Larsen N., Rojas M., Brodie EL., Keller K., Huber T., Dalevi D., Hu P., Andersen GL. 2006. Greengenes, a Chimera-Checked 16S rRNA Gene Database and Workbench Compatible with ARB. Applied and Environmental Microbiology 72:5069-5072. DOI: 10.1128/AEM.03006-05.

Edgar RC. 2010. Search and clustering orders of magnitude faster than BLAST. Bioinformatics 26:2460-2461. DOI: 10.1093/bioinformatics/btq461.

Edgar RC., Haas BJ., Clemente JC., Quince C., Knight R. 2011. UCHIME improves sensitivity and speed of chimera detection. Bioinformatics 27:2194-2200. DOI: 10.1093/bioinformatics/btr381.

Edgar RC. 2013. UPARSE: highly accurate OTU sequences from microbial amplicon reads. Nature Methods 10:996-998. DOI: 10.1038/nmeth.2604.

Eren MA., Maignien L., Sul WJ., Murphy LG., Grim SL., Morrison HG., Sogin ML. 2013. Oligotyping: differentiating between closely related microbial taxa using 16S rRNA gene data. Methods in Ecology and Evolution 4:1111-1119. DOI: 10.1111/2041-210X.12114.

Eren AM., Morrison HG., Lescault PJ., Reveillaud J., Vineis JH., Sogin ML. 2014. Minimum entropy decomposition: Unsupervised oligotyping for sensitive partitioning of high-throughput marker gene sequences. The ISME Journal 9:968-979. DOI: 10.1038/ismej.2014.195.

Franic S., Dolan C V., Boorsboom D., Boomsma DI. 2012. Structural Equation Modeling in Genetics. In: Handbook of Structural Equation Modeling. 
511

512

513

514

515

516

517

518

519

520

521

522

523

524

525

526

527

528

529

530

531

532

533

534

535

536

537

538

539

540

541

542

543

544

545

546

547

Goodrich JK., Waters JL., Poole AC., Sutter JL., Koren O., Blekhman R., Beaumont M., Van Treuren W., Knight R., Bell JT., Spector TD., Clark AG., Ley RE. 2014. Human Genetics Shape the Gut Microbiome. Cell 159:789-799. DOI: 10.1016/j.cell.2014.09.053.

Goodrich JK., Davenport ER., Beaumont M., Jackson MA., Knight R., Ober C., Spector TD., Bell JT., Clark AG., Ley RE. 2016. Genetic Determinants of the Gut Microbiome in UK Twins. Cell Host \& Microbe 19:731-743. DOI: $10.1016 /$ j.chom.2016.04.017.

He Y., Caporaso JG., Jiang X-T., Sheng H-F., Huse SM., Rideout JR., Edgar RC., Kopylova E., Walters WA., Knight R., Zhou H-W. 2015. Stability of operational taxonomic units: an important but neglected property for analyzing microbial diversity. Microbiome 3:20. DOI: 10.1186/s40168-015-0081-x.

Huse SM., Welch DM., Morrison HG., Sogin ML. 2010. Ironing out the wrinkles in the rare biosphere through improved OTU clustering. Environmental Microbiology 12:1889-1898. DOI: 10.1111/j.14622920.2010.02193.x.

Jackson MA., Jeffery IB., Beaumont M., Bell JT., Clark AG., Ley RE., O’Toole PW., Spector TD., Steves CJ. 2016. Signatures of early frailty in the gut microbiota. Genome Medicine 8:8. DOI: 10.1186/s13073-016-0262-7.

Jackson MA., Goodrich JK., Maxan M-E., Freedberg DE., Abrams JA., Poole AC., Sutter JL., Welter D., Ley RE., Bell JT., Spector TD., Steves CJ. 2016. Proton pump inhibitors alter the composition of the gut microbiota. Gut 65:749-756. DOI: 10.1136/gutjnl-2015-310861.

Jones S. 2013. Trends in microbiome research. Nature Biotechnology 31:277-277. DOI: 10.1038/nbt.2546.

Kopylova E., Noe L., Touzet H. 2012. SortMeRNA: fast and accurate filtering of ribosomal RNAs in metatranscriptomic data. Bioinformatics 28:3211-3217. DOI: 10.1093/bioinformatics/bts611.

Kopylova E., Navas-Molina JA., Mercier C., Xu ZZ., Mahé F., He Y., Zhou H-W., Rognes T., Caporaso JG., Knight R. 2016. Open-Source Sequence Clustering Methods Improve the State Of the Art. mSystems 1:e00003-15. DOI: $10.1128 / \mathrm{mSystems.00003-15.}$

Kozich JJ., Westcott SL., Baxter NT., Highlander SK., Schloss PD. 2013. Development of a dual-index sequencing strategy and curation pipeline for analyzing amplicon sequence data on the miseq illumina sequencing platform. Applied and Environmental Microbiology 79:5112-5120. DOI: 10.1128/AEM.01043-13.

Li W., Godzik A. 2006. Cd-hit: a fast program for clustering and comparing large sets of protein or nucleotide sequences. Bioinformatics 22:1658-1659. DOI: 10.1093/bioinformatics/btl158.

Liu Z., DeSantis TZ., Andersen GL., Knight R. 2008. Accurate taxonomy assignments from 16S rRNA sequences produced by highly parallel pyrosequencers. Nucleic Acids Research 36:e120-e120. DOI: 10.1093/nar/gkn491.

Mahé F., Rognes T., Quince C., de Vargas C., Dunthorn M. 2014. Swarm: robust and fast clustering method for amplicon-based studies. PeerJ 2:e593. DOI: 10.7717/peerj.593.

Martin NG., Eaves LJ., Kearsey MJ., Davies P. 1978. The power of the classical twin study. Heredity 40:97-116. DOI: $10.1038 /$ hdy.1978.10.

Mercier C., Boyer F., Bonin A., Coissac E. 2013. SUMATRA and SUMACLUST : fast and exact comparison and clustering of sequences. Abstract In: SeqBio 25-26th Nov 2013:27. 
548

549

550

551

552

553

554

555

556

557

558

559

560

561

562

563

564

565

566

567

568

569

570

571

572

573

574

575

576

577

578

579

580

581

582

583

584

585

586

Mignard S., Flandrois JP. 2006. 16S rRNA sequencing in routine bacterial identification: A 30-month experiment. Journal of Microbiological Methods 67:574-581. DOI: 10.1016/j.mimet.2006.05.009.

Moayyeri A., Hammond CJ., Valdes AM., Spector TD. 2013. Cohort Profile: TwinsUK and Healthy Ageing Twin Study. International Journal of Epidemiology 42:76-85. DOI: 10.1093/ije/dyr207.

Navas-Molina JA., Peralta-Sánchez JM., González A., McMurdie PJ., Vázquez-Baeza Y., Xu Z., Ursell LK., Lauber C., Zhou H., Song SJ., Huntley J., Ackermann GL., Berg-Lyons D., Holmes S., Caporaso JG., Knight R. 2013. Advancing Our Understanding of the Human Microbiome Using QIIME. In: Microbial Metagenomics, Metatranscriptomics, and Metaproteomics. Elsevier Inc., 371-444. DOI: 10.1016/B978-0-12-4078635.00019-8.

Pace NR. 1997. A Molecular View of Microbial Diversity and the Biosphere. Science 276:734-740. DOI: 10.1126/science.276.5313.734.

R Development Core Team. 2009. R: A language and environment for statistical computing. Vienna, Austria: R Foundation for Statistical Computing.

Rognes T., Mahé F., Flouri T., Quince C., Nichols B. 2016. VSEARCH. Available at: https://github.com/torognes/vsearch.

Schloss PD., Handelsman J. 2005. Introducing DOTUR, a Computer Program for Defining Operational Taxonomic Units and Estimating Species Richness. Applied and Environmental Microbiology 71:1501-1506. DOI: 10.1128/AEM.71.3.1501-1506.2005.

Schloss PD., Westcott SL., Ryabin T., Hall JR., Hartmann M., Hollister EB., Lesniewski R a., Oakley BB., Parks DH., Robinson CJ., Sahl JW., Stres B., Thallinger GG., Van Horn DJ., Weber CF. 2009. Introducing mothur: Open-Source, Platform-Independent, Community-Supported Software for Describing and Comparing Microbial Communities. Applied and Environmental Microbiology 75:7537-7541. DOI: 10.1128/AEM.01541-09.

Schloss PD., Gevers D., Westcott SL. 2011. Reducing the Effects of PCR Amplification and Sequencing Artifacts on 16S rRNA-Based Studies. PLoS ONE 6:e27310. DOI: 10.1371/journal.pone.0027310.

Schloss PD. 2016. Application of a Database-Independent Approach To Assess the Quality of Operational Taxonomic Unit Picking Methods. mSystems 1:e00027-16. DOI: 10.1128/mSystems.00027-16.

Schmidt TSB., Matias Rodrigues JF., von Mering C. 2015. Limits to robustness and reproducibility in the demarcation of operational taxonomic units. Environmental Microbiology 17:1689-1706. DOI: 10.1111/14622920.12610 .

Stackebrandt E., Goebel BM. 1994. Taxonomic Note: A Place for DNA-DNA Reassociation and 16S rRNA Sequence Analysis in the Present Species Definition in Bacteriology. International Journal of Systematic and Evolutionary Microbiology 44:846-849. DOI: 10.1099/00207713-44-4-846.

Visscher PM. 2004. Power of the Classical Twin Design Revisited. Twin Research 7:505-512. DOI: 10.1375/twin.7.5.505.

Westcott SL. 2016.Mothur - Version 1.37.0. Available at https://github.com/mothur/mothur/releases/tag/v1.37.0 (accessed April 20, 2016).

Westcott SL., Schloss PD. 2015. De novo clustering methods outperform reference-based methods for assigning 16S rRNA gene sequences to operational taxonomic units. PeerJ 3:e1487. DOI: 10.7717/peerj.1487.

Peer] reviewing PDF | (2016:06:11085:1:1:NEW 16 Jul 2016) 


\section{Figure 1}

Twin based A, C, and E estimate comparisons between closed and open reference, and de novo clustering using UCLUST with a similarity threshold of $97 \%$.

A) Boxplots representing the A, C and E estimates for all OTUs found in at least $50 \%$ of samples in each method. De novo clustering A estimates significantly higher than those of closed reference clustering $(q=0.017) B)$ The same estimates as in A but displayed as a density function showing the distribution of estimates amongst OTUs. 
A

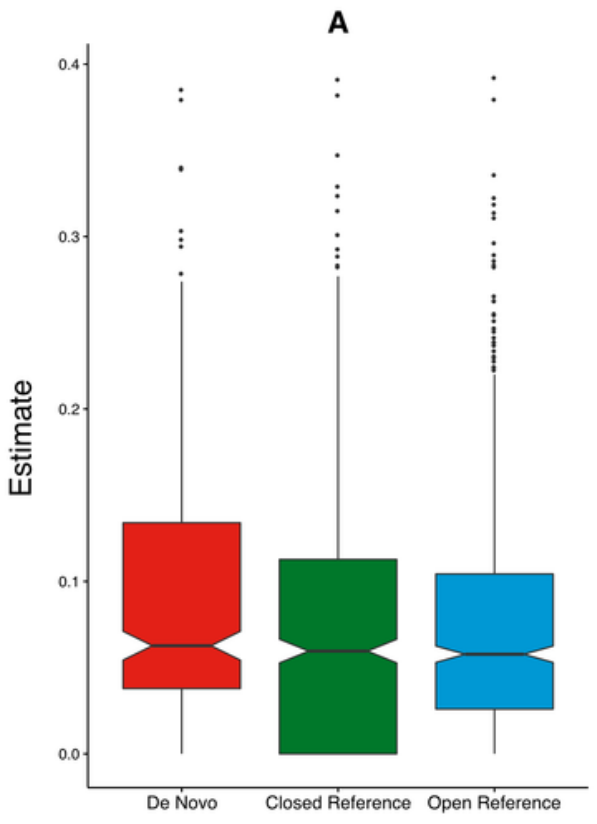

B

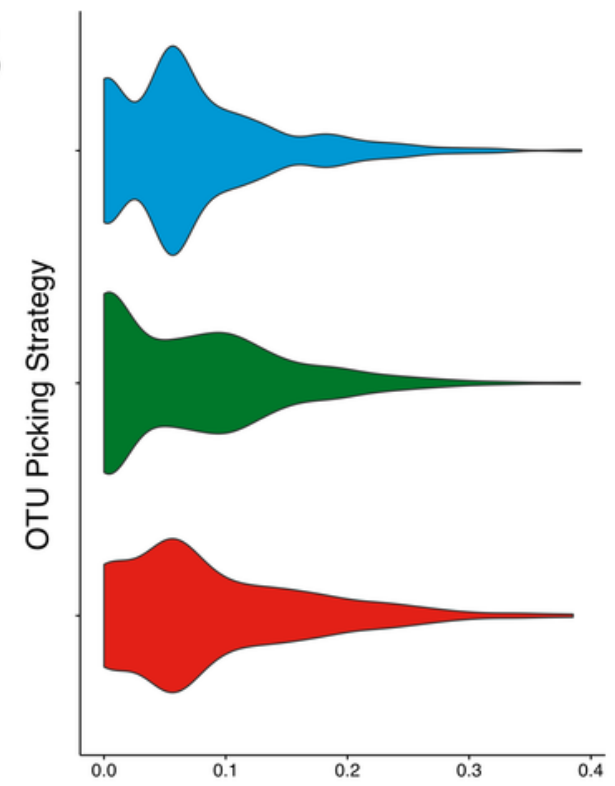

C
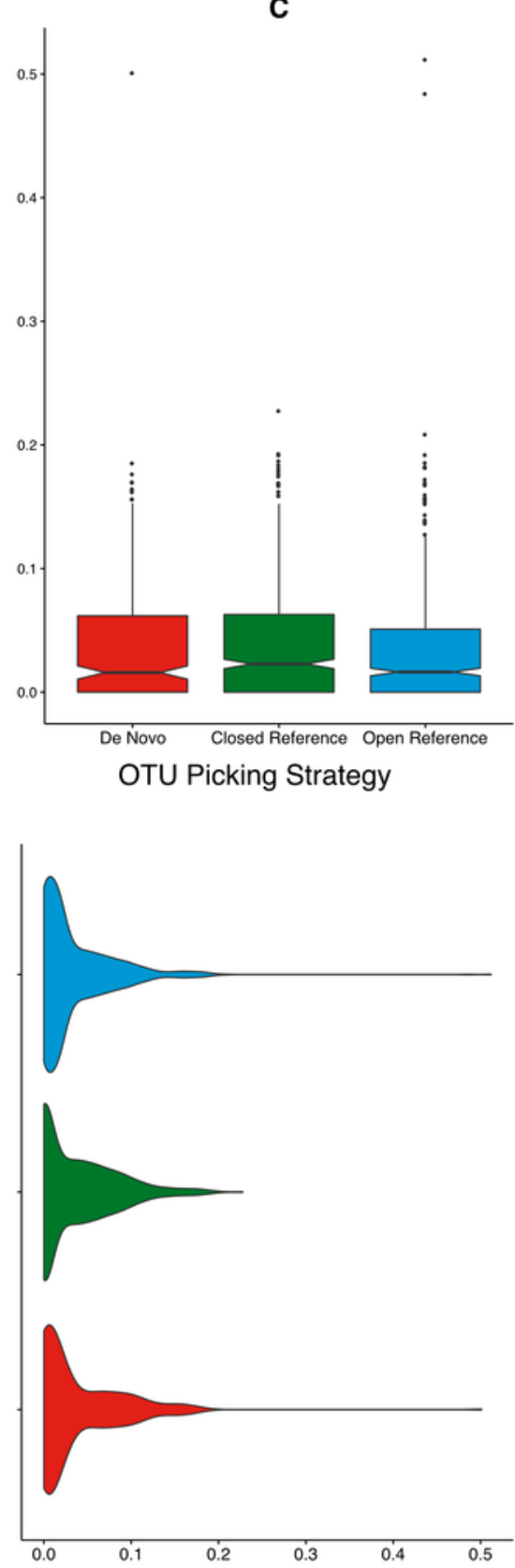

Heritability Estimate
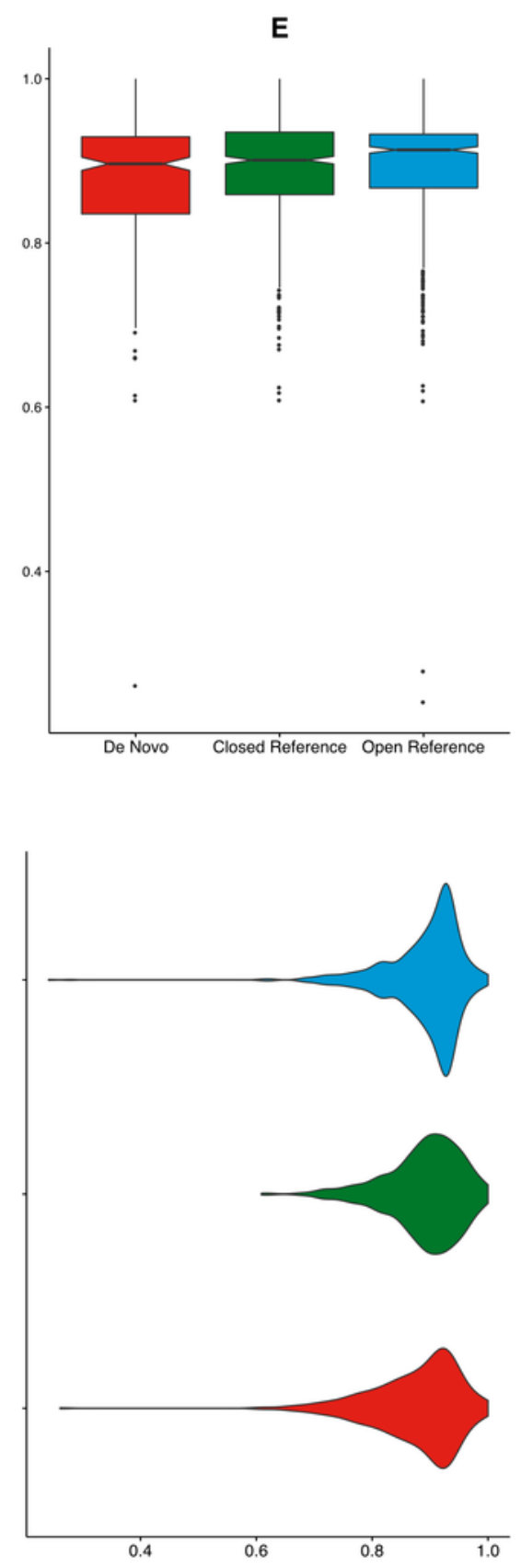


\section{Figure 2}

Twin based A, C, and E estimate comparisons between different greedy algorithms for de novo clustering at a $97 \%$ similarity threshold

A) Boxplots representing the A, C and E estimates for all OTUs found in at least $50 \%$ of samples in each method. There was no significant difference in A estimates between methods. B) The same estimates as in A but displayed as a density function showing the distribution of estimates amongst OTUs. 
A

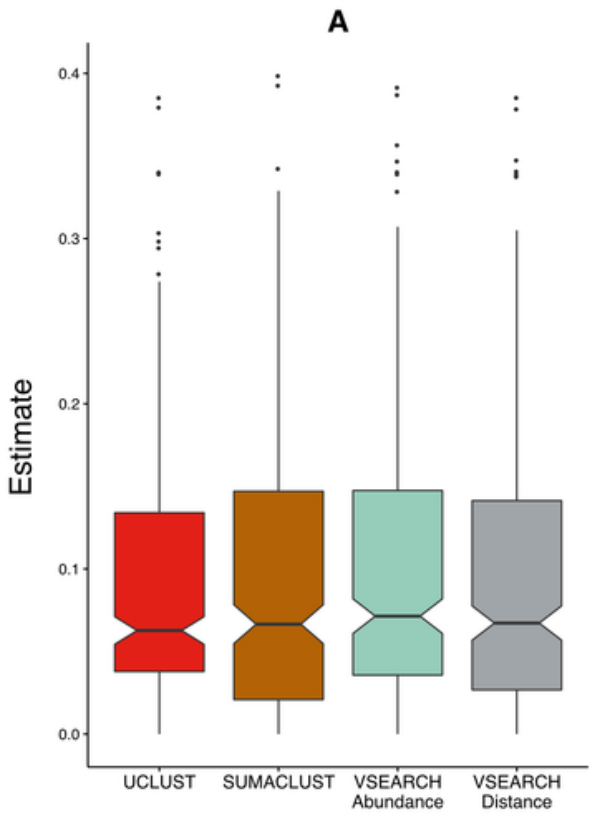

B

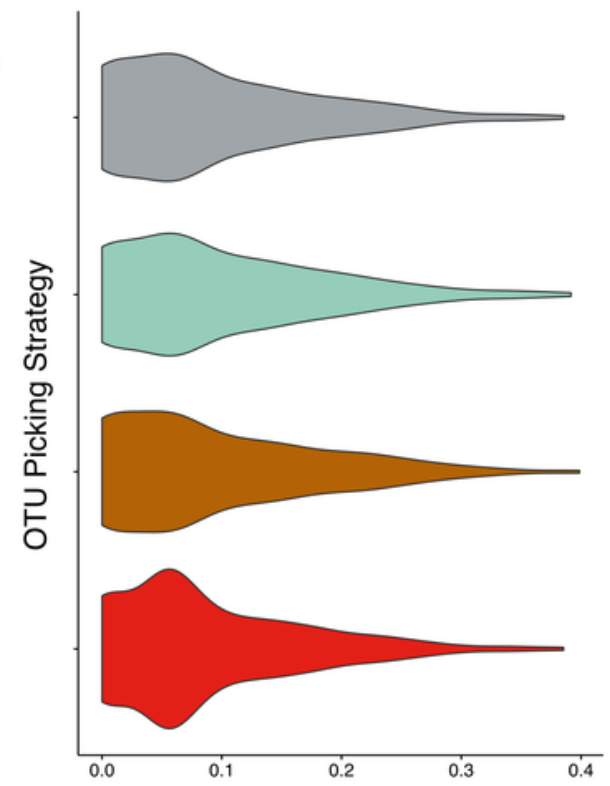

C
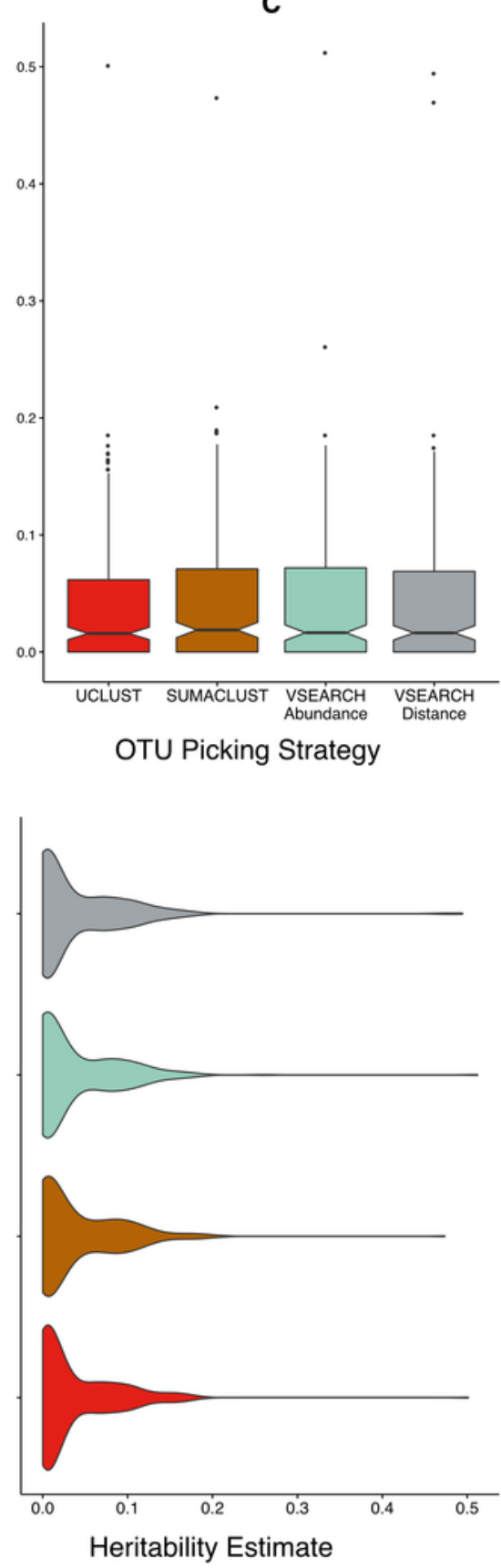

E
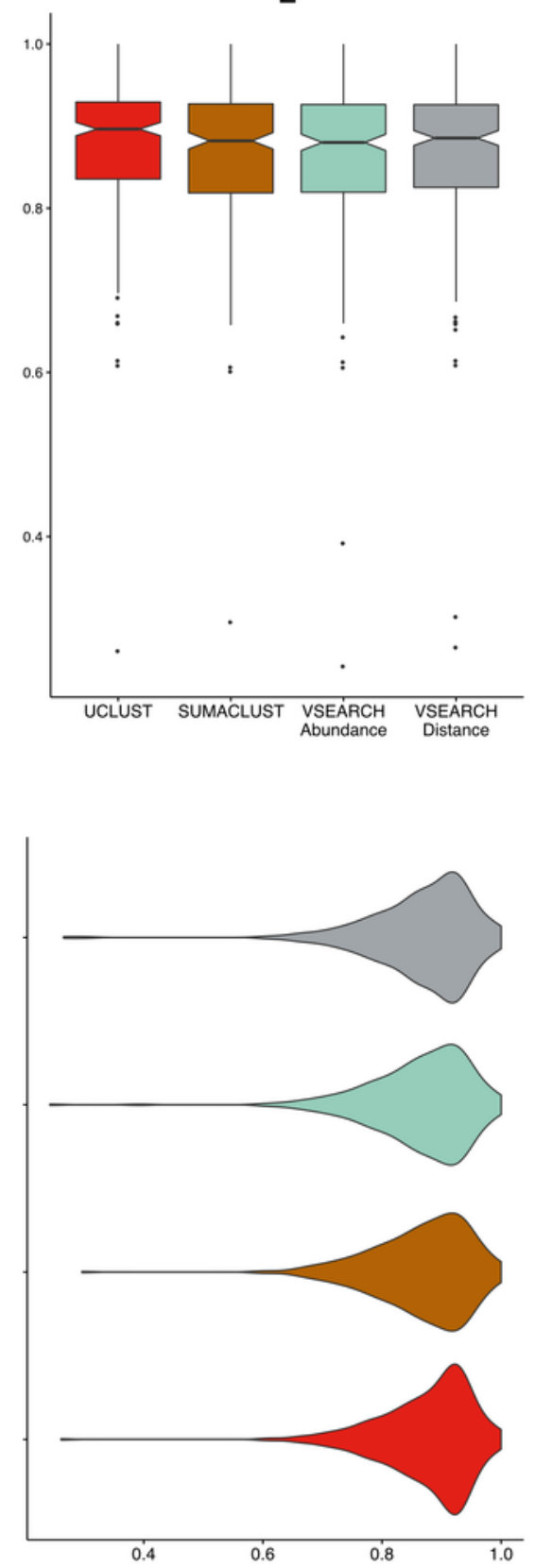


\section{Figure 3}

Twin based A, C, and E estimate comparisons between three different thresholds of de novo clustering using VSEARCH, VSEARCH de-replicated sequences, and two nonthreshold based techniques.

A) Boxplots representing the A, C and E estimates for all OTUs found in at least $50 \%$ of samples in each method. The $97 \%$ threshold produced significantly more higher A estimates than the 99 and $100 \%$ thresholds $(q=0.02, q=0.0001)$. B) The same estimates as in A but displayed as a density function showing the distribution of estimates amongst OTUs.

A

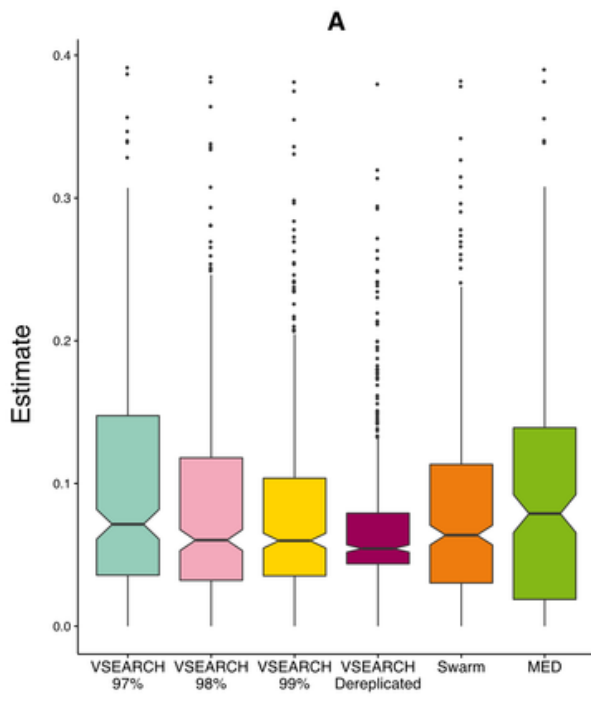

B

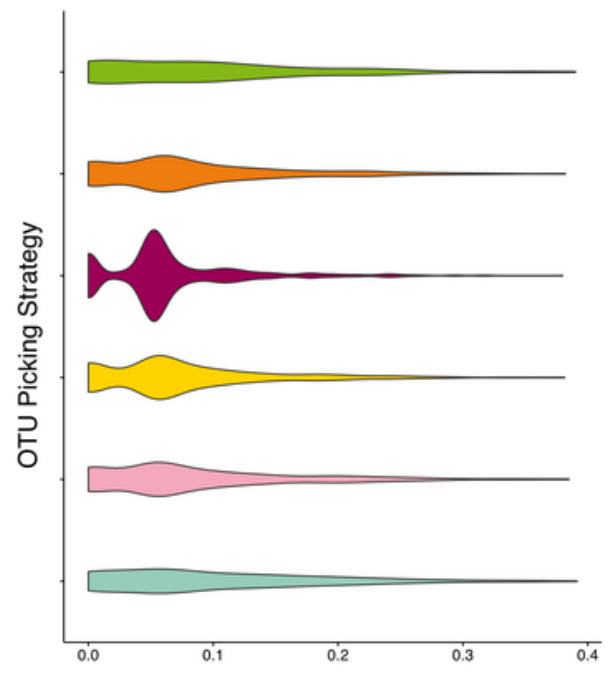

C
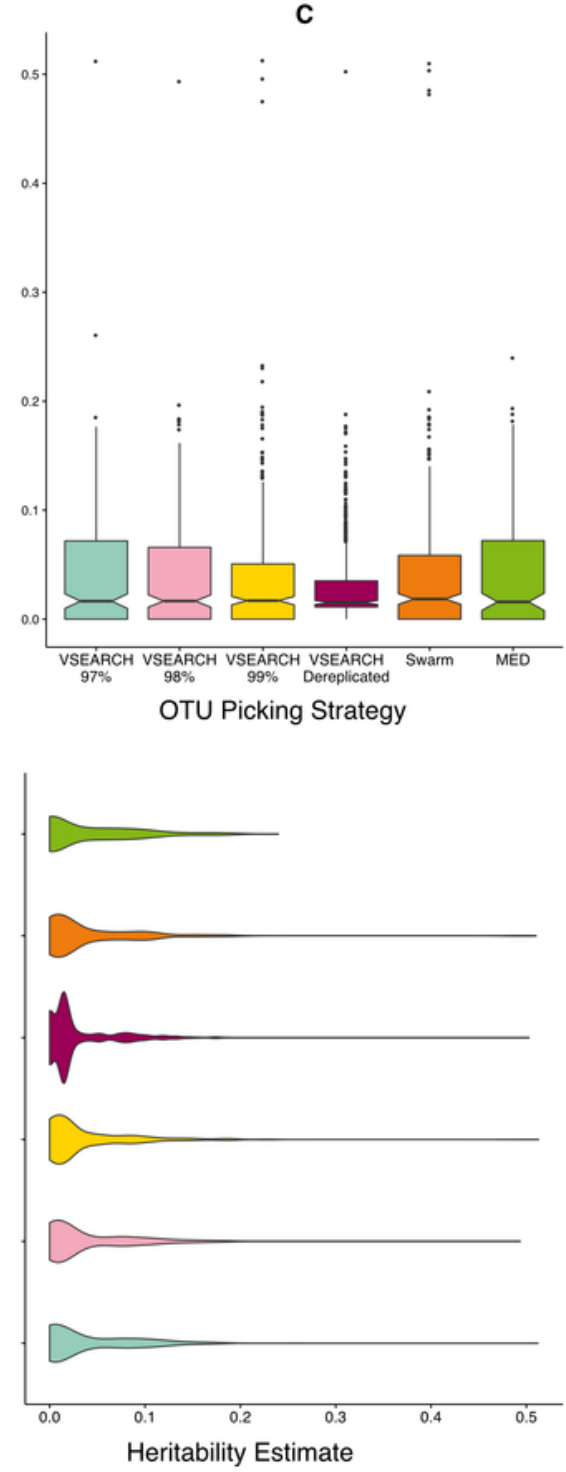

E
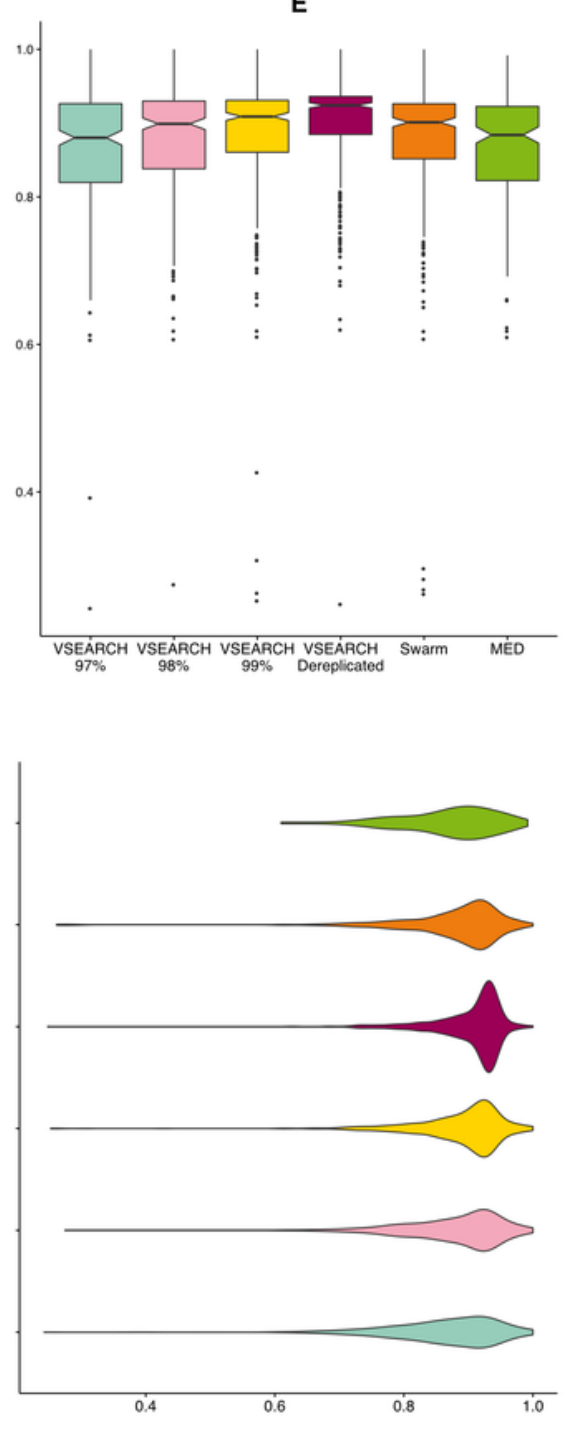
Figure 4

Comparison of $A$ heritability estimates between all clustering approaches. Only considering OTUs who's A estimate was greater than the mean $(\sim 8 \%)$ and had a lower $95 \% \mathrm{Cl}$ greater than $1 \%$.

SUMACLUST and VSEARCH clustering produced OTUs with significantly higher heritability estimates than OTUs produced using reference-based clustering. Significant differences are shown where $*$ indicates $q<0.05$ and $* *$ indicates $q<0.01$.

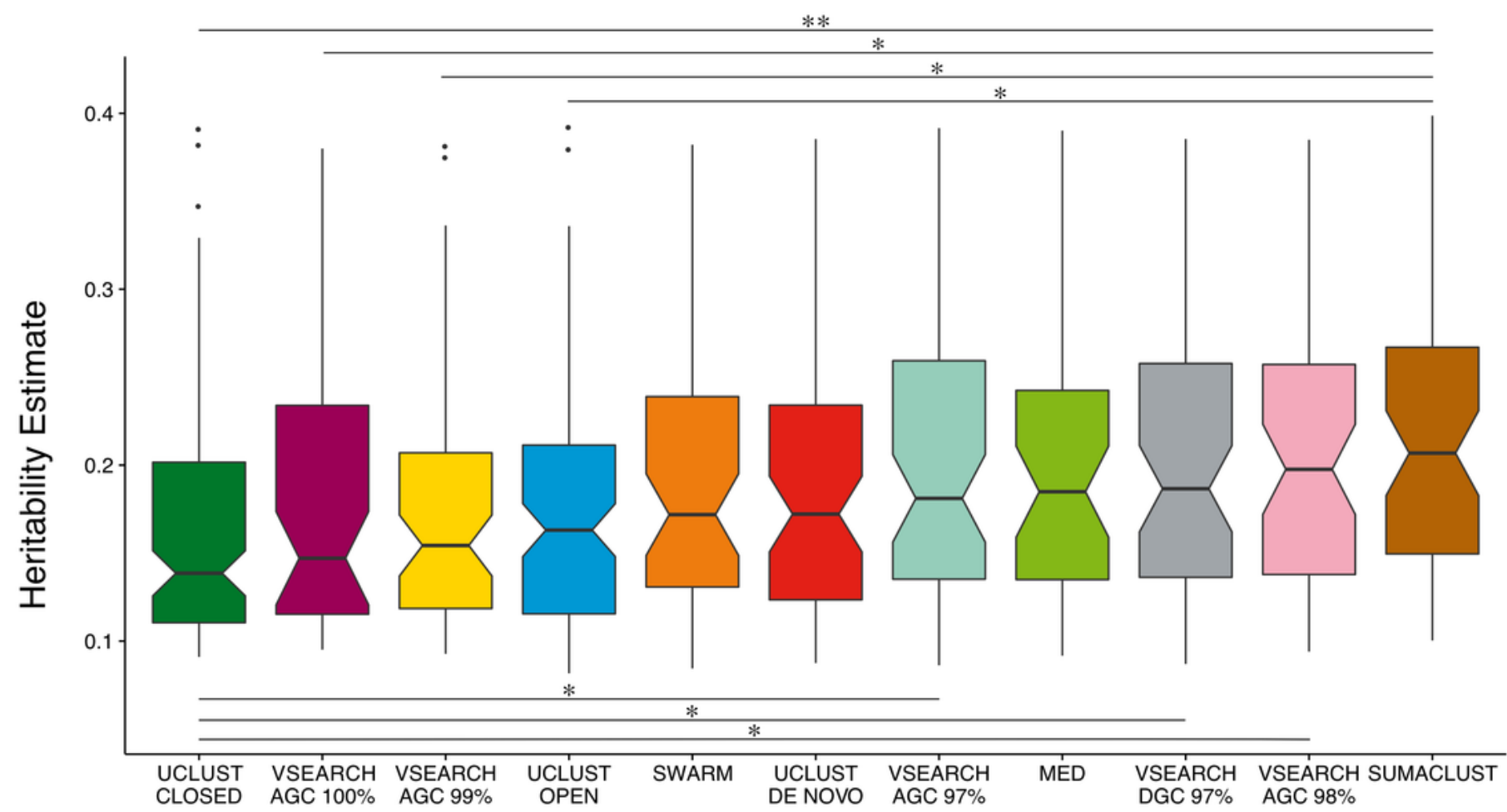

Clustering Approach 
Figure 5

Comparison of absolute alpha diversity values for Shannon, Simpson, Chaol, and OTU count indices across all samples.

OTU tables for each method were rarefied to 10000 sequences 25 times and the mean diversity calculated across all tables. There was a significant difference in the distribution of diversity values between all methods for all four metrics. De-replicated sequences in particular inflate richness-based measures.
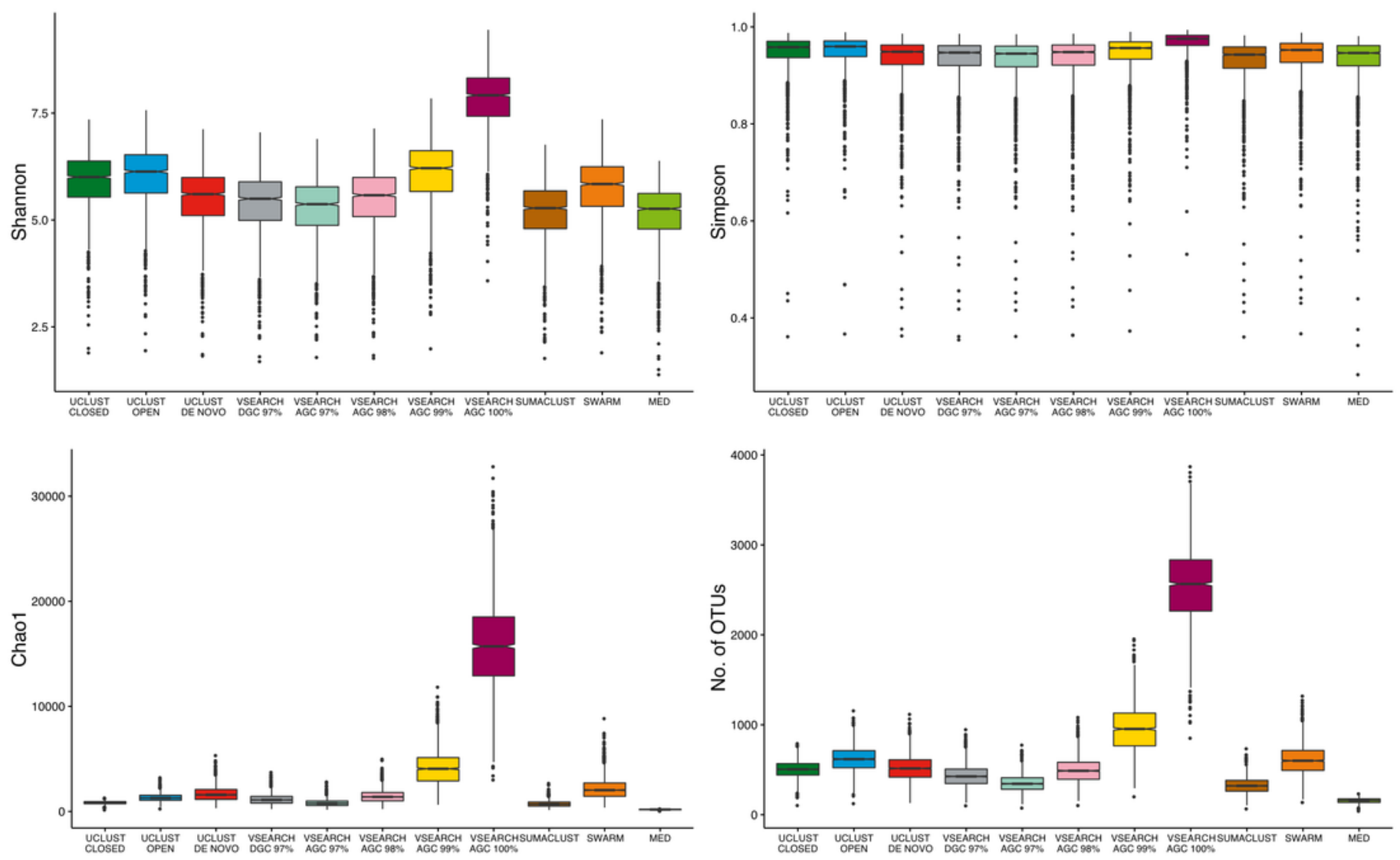


\section{Figure 6}

Kendall's Tau rank based correlations between samples across methods for each of Shannon, Simpson, Chaol and OTU count metrics.

Rank correlation represents the concordance between relative diversity assignments between the same samples in each clustering method. There is generally high correlation between all methods when using the Shannon and Simpson indices, which measure evenness of species distribution. However, the de-replicated, closed reference, and MED clustered OTUs show poor correlation in the richness measures (Chaol and OTU count). Clustering method may therefore influence diversity association analyses. 


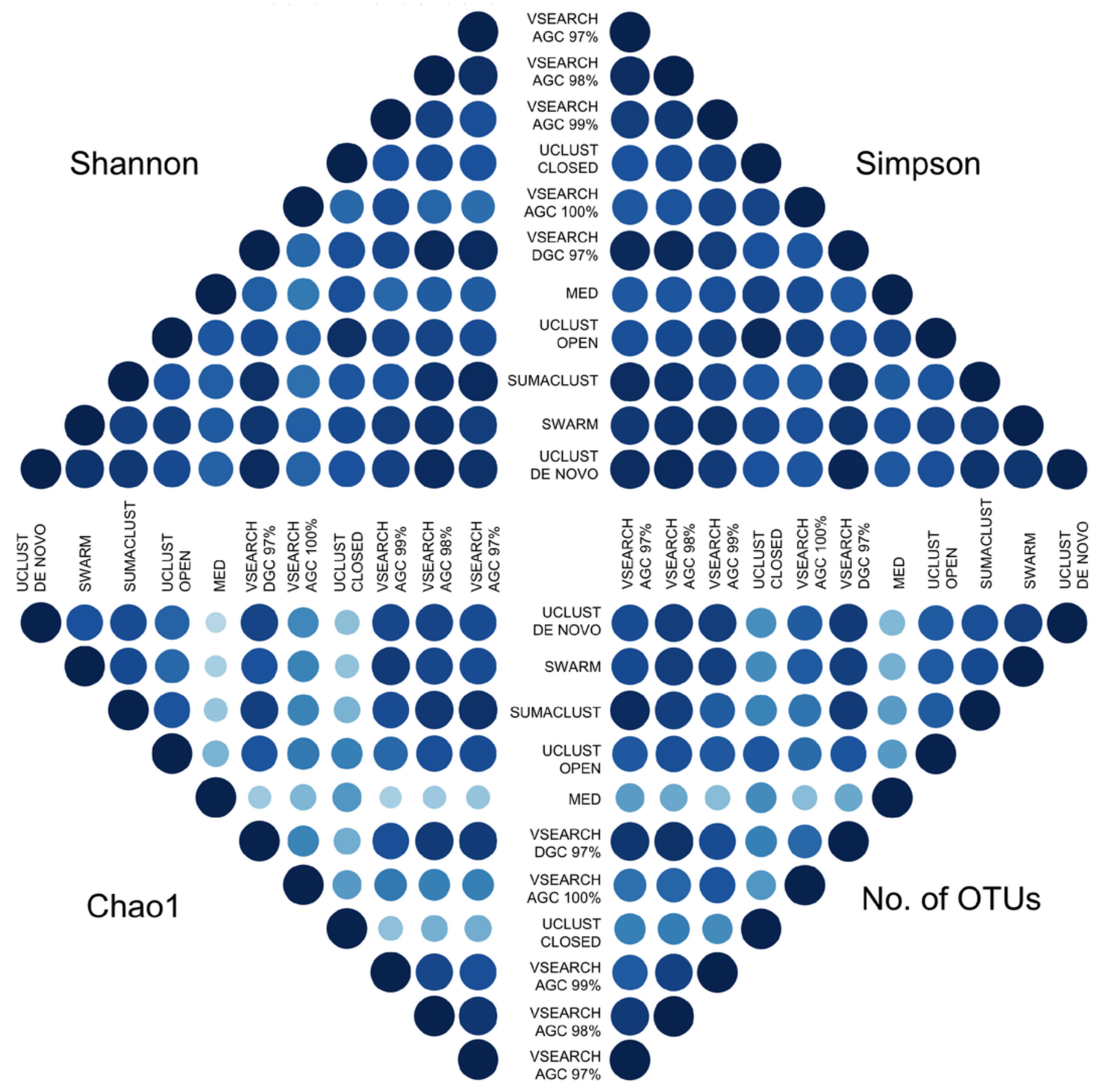

Kendall rank correlation coefficient

\begin{tabular}{lllllllllll|}
\hline-1 & -0.8 & -0.6 & -0.4 & -0.2 & 0 & 0.2 & 0.4 & 0.6 & 0.8 & 1
\end{tabular}

